Çukurova Üniversitesi Mühendislik Mimarlık Fakültesi Dergisi, 31(ÖS 2), ss. ÖS 161-ÖS 167, Ekim 2016

Cukurova University Journal of the Faculty of Engineering and Architecture, 31(SI 2), pp. SI 161-SI 167, October 2016

\title{
Tamamlayıcı Fonksiyonlar Yöntemi ile Heterojen Bir Çubuğun Zorlanmış Titreşim Analizi
}

\author{
Kerimcan ÇELEBİ ${ }^{* 1}$, Durmuş YARIMPABUÇ ${ }^{2}$, Mehmet EKER ${ }^{3}$ \\ ${ }^{1}$ Adana Bilim ve Teknoloji Üniversitesi, Mühendislik ve Doğa Bilimleri Fakültesi, Makine \\ Mühendisliği Bölümü, Adana \\ ${ }^{2}$ Osmaniye Korkut Ata Üniversitesi, Fen Edebiyat Fakültesi, Matematik Bölümü, Osmaniye \\ ${ }^{3}$ Osmaniye Korkut Ata Üniversitesi, Mühendislik Fakültesi, Makine Mühendisliği Bölümü, \\ Osmaniye
}

Geliş tarihi: 01.09.2016 Kabul tarihi: 02.10.2016

\section{$\ddot{O} z$}

Sürekli sistem olarak modellenen eksenel yüklenmiş heterojen bir çubuğun elastik davranış problemi analiz edilmiştir. Bu problemi modelleyen diferansiyel denklemlere Laplace dönüşümü uygulanarak zamandan bağımsız sınır değer problemi eksenel koordinatlarda elde edilmiş daha sonra bu problem tamamlayıcı fonksiyonlar metodu (TFM) tarafindan çözülmüştür. Sayısal olarak çözülen denklemler Durbin'in sayısal ters dönüşümünü yardımıyla zaman uzayına dönüştürülmüştür. Her bir yükleme tipi ve inhomojenlik parametresi için elde edilen sayısal sonuçlar, analitik sonuçlar ve ANSYS sonuçları ile karşılaştırılmıştır. Bu birleşik yöntem, iyi yapılandırılmış, basit ve etkili bir yöntemdir.

Anahtar Kelimeler: Heterojen çubuk, Zorlanmış titreşim, Laplace, Tamamlayıcı fonksiyonlar yöntemi

\section{Forced Vibration Analysis of A Heterogeneous Rod by Complementary Functions Method}

\begin{abstract}
The axial vibration problem formulation and solution of a heterogeneous rod modelled as a continuous system were analyzed. By applying Laplace transformation to the differential equations that model to this problem, time independent boundary value problems were obtained in the axial coordinates, then this problem is solved by the complementary functions method. The equations solved numerically is converted into time space with the help of Durbin's numerical inverse transformation. The numerical results that obtained for each load type and inhomogeneity parameter were compared with analytical and ANSYS results in the literature. This unified method is well-structured, simple and efficient.
\end{abstract}

Keywords: Heterogeneous rod, Forced vibration, Laplace, Complementary functions method

\footnotetext{
*Sorumlu yazar (Corresponding author): Kerimcan ÇELEBİ, kcancelebi@adanabtu.edu.tr
} 


\section{GíRiş}

Malzeme mühendisliği alanındaki gelişmeler, bazı tür ihtiyaçların karşılanabilmesi için malzemelerde homojen olmayan kademeli veya devamlı değişen yap1 elemanlarının imalatını mümkün kılmıştır. Tasarımcı tarafindan heterojen yapıya sahip elemanların kullanılması ağırlığın azaltılmasında yardımc1 olduğu gibi yapıların dayanımını, tokluğunu ve kararlılığını da arttırmaktadır. Heterojen yapıya sahip çubukların dinamik yük altındaki davranışlarının analizi, kompozit malzeme araştırmalarında önemli bir yer tutmaktadır. Dinamik eksenel yük altındaki heterojen yapıya sahip çubukların analizi tek boyutlu titreşim problemleri başlığı altında incelenebilir [1-3]. Bu konularda yapılan çok sayıda çalışma, serbest titreşim analizi ve zorlanmış titreşimin analizlerinde, ağırlıklı olarak, sonlu elemanlar yöntemi ve benzeri nümerik yöntemleri ihtiva etmektedir [4-11].

$\mathrm{Bu}$ çalışmada, heterojen yapıya sahip bir çubuğun uç noktasına dinamik yükler uygulanarak analiz yapılmıştır. Problemi ifade eden diferansiyel denklem, değişken katsayılara sahip olacağından her heterojen yapıyı ifade eden form tipi için mevcut yöntemlerle analitik çözüm pratik olmayacaktır. Bundan dolayı, analizde Laplace transformasyon yöntemi ile birlikte Tamamlayııı fonksiyonlar metodu (TFM) kullanılmıştır. Laplace transformasyon yöntemi zamandan bağımsız sınır değer problemini eksenel koordinatlarda verirken daha sonra bu problem TFM tarafindan çözülmektedir. TFM benzer problemleri başlanğıç-değer sistemine çevirerek literatürdeki herhangi bir sayısal yöntem ile çözülmesine izin vermektedir. $\mathrm{Bu}$ çalışmada beşinci dereceden Runge-Kutta metodu kullanılarak sayısal olarak çözülen denklem sistemi Durbin'in sayısal ters dönüşümünü kullanarak zaman uzayına dönüştürülmüştür. Metodun teorisi literatürde [12-14] mevcuttur. Bu yöntem ayrıca çubuk harici elemanların kullanıldığı diğer bazı yapısal mekanik problemlerinde de başarılı bir şekilde uygulanmıştır [15-17]. Çubuğun serbest ucuna ait deplasman değerleri Çizelgelar halinde verilmiş, elde edilen zorlanmış analiz sonuçları literatürdeki analitik sonuçlar Çelebi [10] ve sonlu elemanlar yöntemi kullanarak sayısal çözüm yapan ANSYS paket program sonuçları ile karşılaştırılmıştır. TFM sonuçlarının diğer bir sayısal çözüm yapan ANSYS sonuçlarına göre analitik sonuçlara çok daha yaklaştığı gözlemlenmiştir. Tamamlayıcı fonksiyonlar metodunun çubuğun dinamik analizinde sağladığı diğer avantajlar ise (1) zorlanmış titreşim etkisi doğrudan elde edilir, (2) zorlanmış titreşim analizi için serbest titreşim frekanslarına ve mod şekillerine ihtiyaç yoktur, (3) çözüm yöntemi heterojen yapıyı ifade eden belirli bazı fonksiyonlara bağımlı değildir, heterojen yapıyı ifade eden tüm keyfi fonksiyonlar için son derece uygundur.

\section{TEORI}

Eksenel yük altında koordinatına göre yoğunluğu $\rho(\mathrm{x})$ ve elastisite modülü $\mathrm{E}(\mathrm{x})$ değişen sabit kesit alanına sahip bir çubuğun davranışı aşağıdaki diferansiyel denklem ile verilir [10].

$\frac{\partial^{2} u(x, t)}{\partial x^{2}}+\frac{1}{E(x)} \frac{\partial E(x)}{\partial x} \frac{\partial u(x, t)}{\partial x}=\frac{\rho(x)}{E(x)} \frac{\partial^{2} u(x, t)}{\partial t^{2}}$

Denklem (1)'i boyutsuz olarak ifade etmek istersek

$\frac{\partial^{2} v(\eta, \tau)}{\partial \eta^{2}}+\frac{1}{E(\eta)} \frac{\partial E(\eta)}{\partial \eta} \frac{\partial v(\eta, \tau)}{\partial \eta}=c^{2} \frac{\rho(\eta)}{E(\rho)} \frac{\partial^{2} v(\eta, \tau)}{\partial \tau^{2}}$

şeklinde olur. Burada $c^{2}=E / \rho$ olup, "c" deplasman yayılma hızıdır. Bu işlem için aşağıdaki boyutsuz değişkenler kullanılmıştır.

$v(\eta, \tau)=\frac{u(x, t)}{L}, \quad \eta=\frac{x}{L}, \quad \tau=\frac{c t}{L}$

Çubuğun uçları sabit-serbest şeklindedir. $\eta=0$ ucunda ankastre olan çubuk başlangıçta hareketsizdir. $P(\tau)$ kuvveti $\eta=1,0$ 'deki serbest uca eksenel yönde uygulanmaktadır.

Sınır şartları,

$v(\eta, 0)=0, \frac{\partial v(\eta, 0)}{\partial \tau}=0$ 
$v(0, \tau)=0, \frac{\partial v(1, \tau)}{\partial \eta}=\frac{P(\tau)}{A(1) E(1)}$

şeklinde elde edilir. Denklem (2), yoğunluk $\rho(\eta)$ ve elastisite modülü $E(\eta)$ 'nin formuna bağlı olarak, değişken katsayılı bir denklem olduğundan genel çözümü yoktur. Analitik olarak özel bazı $\rho(\eta)-E(\eta)$ formları için çözülebilirken sayısal olarak ise tamamlayıc1 fonksiyonlar metodu kullanılarak yoğunluk ve elastisite modülünü ifade eden tüm keyfi fonksiyonlar için çözüm elde edilebilir. Çalışmamızda analitik çözüm ile sayısal çözümleri karşılaştırabilmemiz adına bu formlar $\sin ^{2}[a \eta+b], \quad(1+a \eta)^{2}$ ve $e^{-a \eta}$ olarak tespit edilmiştir. Çubuğun serbest ucuna uygulanacak kuvvetler ise $\mathrm{P}_{1}(\tau)=\mathrm{P}_{0}(1-\cos [\gamma \tau)], \quad \mathrm{P}_{2}(\tau)=\mathrm{P}_{0}$ ve $\mathrm{P}_{3}(\tau)=\mathrm{P}_{0}\left(1-\mathrm{e}^{-\gamma \tau}\right)$ olarak düşünülmüştür.

\section{1. $E(\eta)=E_{0}(1+a \eta)^{2}$ ve $\rho(\eta)=\rho_{0}(1+a \eta)^{2}$ İfadeleri İçin Çözüm}

Yoğunluk ve elastisite ifadelerinin boyutsuz büyüklük olan $\eta$ ile $E(\eta)=E_{0}(1+a \eta)^{2}$ ve $\rho(\eta)=\rho_{0}(1+a \eta)^{2}$ şeklinde değiştiğini, kesit alanının $\mathrm{A}(\eta)=\mathrm{A}_{0}$ ise üniform olduğunu kabul edersek diferansiyel denklem ve sınır şartları Laplace uzayında

$y^{11}(\eta, s)+\frac{2 a}{(1+a \eta)} y^{1}(\eta, s)-s^{2} y(\eta, s)=0$

$\mathrm{y}(0, \mathrm{~s})=0, \frac{\partial \mathrm{y}(1, \mathrm{~s})}{\partial \eta}=\frac{\mathrm{L}\{\mathrm{P}(\tau)\}}{\mathrm{E}_{0}(1+\mathrm{a})^{2} \mathrm{~A}_{0}}$

formunu alır. Burada $y(\eta, p)=L\{v(\eta, \tau\}$ ve $s$ ise kompleks Laplace parametresini ifade eder. $\mathrm{Bu}$ safhada, denklem (6)'nın kapalı form çözümü bu ifadeler için değişken dönüşümü yapılarak mümkündür fakat pratik değildir. Dolayısıyla Laplace uzayındaki sonuçların eldesi için TFM kullanılabilir. TFM, ikinci mertebeden sınır-değer problemlerini başlangıç değer-problemine dönüştürme esasına dayanmaktadır. TFM ile denklem (6)'nın genel çözümü $\mathrm{y}=\mathrm{b}_{\mathrm{i}} \mathrm{y}_{\mathrm{i}}, \mathrm{i}=1,2$

şeklindedir, burada $y_{i}$ lineer bağımsız homojen çözüm iken $b_{i}$ sınır şartlarından elde edilecek sabitlerdir.

TFM ile çözüme $y_{i}=Z_{1}^{(i)}$ ve $y_{i}^{1}=Z_{2}^{(i)}$ kabulleri yapılarak başlanır ve

$\left(Z_{1}^{(i)}\right)^{1}=Z_{2}^{(i)}$

$\left(Z_{2}^{(i)}\right)^{1}=-\left(\frac{2 a}{(1+a \eta)}\right) Z_{2}^{(i)}+s^{2} Z_{1}^{(i)}$

eşitlikleri elde edilir. Yukardaki homojen denklem sisteminin çözümü keyfi başlangıç koşulları için sayısal olarak yapılır. Bu keyfi başlangıç koşulları $\mathrm{C}_{\mathrm{ij}}$,

$\mathrm{Z}_{\mathrm{j}}^{(\mathrm{i})}=\mathrm{C}_{\mathrm{ij}}, \mathrm{i}=1,2$

çözümün lineer bağımsızlığını sağlamak için lineer bağımsız olarak seçilmelidir. Bu başlangıç değer problemi beşinci dereceden Runge-Kutta metodu (RK5) ile çözülmüştür. Çözümde $0 \leq \eta \leq 1$ aralığ1 boyunca 21 düğüm noktası (20 aralık) alınmıştır.

$\mathrm{Bu}$ çözüm sonucunda elde edilen $\mathrm{y}_{\mathrm{i}}$ ve türevi $y_{i}^{1}$ 'nin değerleri denklem (7)'de yerine yazılarak bilinmeyen $b_{i}$ katsayılarını içeren iki denklem elde edilir. Bu denklemler matrix formunda

$\left[\begin{array}{cc}\mathrm{C}_{11} & \mathrm{C}_{21} \\ \mathrm{y}_{1}^{1}(1, \mathrm{~s}) & \mathrm{y}_{2}^{1}(1, \mathrm{~s})\end{array}\right]\left[\begin{array}{l}\mathrm{b}_{1} \\ \mathrm{~b}_{2}\end{array}\right]=\left[\begin{array}{c}0 \\ \frac{\mathrm{L}\{\mathrm{P}(\tau)\}}{\mathrm{E}_{0}(1+\mathrm{a})^{2} \mathrm{~A}_{0}}\end{array}\right]$

şeklinde yazılır. Burada, $C_{11}$ ve $C_{21}$ değerleri sırasıyla $\mathrm{y}_{1}(0, \mathrm{~s})$ ve $\mathrm{y}_{2}(0, \mathrm{~s})$ 'e karşılık gelir. $\mathrm{Bu}$ sistem çözülerek $b_{i}$ katsayıları bulunur.

$\mathrm{Bu}$ katsayılar bulunduktan sonra boyutsuz deplasman $\mathrm{v}(\eta, \tau)$ değerlerinin zaman uzayına dönüşümü ters Laplace dönüşümü ile yapılır. Bu amaçla Fast Fourier dönüşümüne (FFT) dayalı modifiye Durbin ters Laplace sayısal dönüşümü kullanılmıştır [18]. 


\section{SAYISAL SONUÇLAR}

$\mathrm{Bu}$ çalışmada sürekli sistem olarak modellenen eksenel yüklenmiş heterojen yapıya sahip bir çubuğun elastik davranış problemi analiz edilmiştir. Bu problemi modelleyen diferansiyel denklemlere Laplace dönüşümü uygulanarak zamandan bağımsız sınır değer problemi eksenel koordinatlarda elde edilmiş daha sonra bu problem TFM tarafindan çözülmüştür. Sayısal olarak çözülen denklemler Durbin'in sayısal ters dönüşümü yardımıyla zaman uzayına dönüştürülmüştür. Çubuğun serbest ucuna etki eden üç tip eksenel dinamik yükleme analizlerde kullanılmıştır: $\mathrm{P}_{1}(\tau)=\mathrm{P}_{0}(1-\cos [\gamma \tau)], \quad \mathrm{P}_{2}(\tau)=\mathrm{P}_{0}$ ve $\mathrm{P}_{3}(\tau)=\mathrm{P}_{0}\left(1-\mathrm{e}^{-\gamma \tau}\right)$. Hesaplamalarda $\gamma$ değeri 0,6 olarak kabul edilmiştir. Yoğunluk ve elastisite ifadelerinde yer alan $a$ inhomojenlik parametresidir ve hesaplamalarda sirasiyla $0,1,2$ değerlerini almakta iken $b$ ise 1 değerini almaktadır. TFM kullanılarak bulunan, çubuğun serbest ucuna ait deplasman sonuçları, literatürdeki analitik sonuçlar [10] ve sonlu elemanlar yöntemini kullanarak sayısal çözüm yapan ANSYS paket programı sonuçları ile karşılaştırılmıştır. ANSYS paket programı ile çözümde yükleme şeklinden dolayı Newmark metodu tercih edilmiştir. Eleman tipi olarak BEAM188 kullanılmış, eksenel yönde değişen kesit ifadesi ANSYS parametrik tasarım dili (APDL) kullanılarak verilmiştir. Sonuçlar belirlenen inhomojenlik parametreleri ve yükleme tipleri için Çizelge 1-9'da sunulmuştur. Çözümlerde, ANSYS'te çubuk eksenel yönde eşit uzunlukta 1000 parçaya bölünerek çözüm yapılırken TFM'de çubuk 20 parçaya bölünerek çözüm elde edilmiştir. TFM sonuçları ile analitik sonuçların, ANSYS sonuçlarına göre birbirine daha yakın çıktığı gözlemlenmiştir.

Çizelge 1. Yoğunluğu ve elastisite modülü üstel formda değişen çubuk için $P_{1}(\tau)$ yükü altında TFM ile bulunan serbest uçtaki deplasman değerlerinin analitik ve ANSYS sonuçları ile karşılaştırılması

\begin{tabular}{|c|c|c|c|c|c|c|c|c|c|}
\hline \multicolumn{10}{|c|}{$v(1, \tau)$} \\
\hline & \multicolumn{3}{|c|}{$a=0$} & \multicolumn{3}{|c|}{$a=1$} & \multicolumn{3}{|c|}{$a=2$} \\
\hline$\tau$ & TFM & Ref. [10] & ANSYS & TFM & Ref. [10] & ANSYS & TFM & Ref. [10] & ANSYS \\
\hline 5 & 2,13294 & 2,133376 & 2,12915 & 3,43205 & 3,432317 & 3,42647 & 6,57568 & 6,577714 & 6,57017 \\
\hline 10 & $-0,23283$ & $-0,23388$ & $-0,23456$ & 0,067883 & 0,06748 & 0,065364 & $-0,12812$ & $-0,13023$ & $-0,13670$ \\
\hline 20 & 0,179831 & 0,179786 & 0,176996 & 0,276992 & 0,276589 & 0,275055 & 0,425383 & 0,424095 & 0,422169 \\
\hline 30 & 0,1105 & 0,109508 & 0,108413 & 0,613708 & 0,613464 & 0,610443 & 1,04217 & 1,04133 & 1,02593 \\
\hline 40 & 0,659759 & 0,659849 & 0,654500 & 1,03681 & 1,036546 & 1,03251 & 1,67432 & 1,672538 & 1,66657 \\
\hline 50 & 0,688788 & 0,688 & 0,686527 & 1,51709 & 1,516876 & 1,51444 & 2,84346 & 2,843814 & 2,82331 \\
\hline
\end{tabular}

Çizelge 2. Yoğunluğu ve elastisite modülü üstel formda değişen çubuk için $P_{2}(\tau)$ yükü altında TFM ile bulunan serbest uçtaki deplasman değerlerinin analitik ve ANSYS sonuçları ile karşı1laştırılması

\begin{tabular}{|c|c|c|c|c|c|c|c|c|c|}
\hline \multicolumn{10}{|c|}{$v(1, \tau)$} \\
\hline & \multicolumn{3}{|c|}{$\mathbf{a}=\mathbf{0}$} & \multicolumn{3}{|c|}{$a=1$} & \multicolumn{3}{|c|}{$a=2$} \\
\hline$\tau$ & TFM & Ref, [10] & ANSYS & TFM & Ref, [10] & ANSYS & TFM & Ref, [10] & ANSYS \\
\hline 5 & 1,00014 & 1,000407 & 0,982324 & 2,82698 & 2,829704 & 2,85501 & 2,20688 & 2,193008 & 2,30300 \\
\hline 10 & 1,95421 & 1,978580 & 1,90338 & 0,706242 & 0,750206 & 0,682098 & 4,27086 & 4,344166 & 4,46342 \\
\hline 20 & 0,0508049 & 0,0264 & 0,115879 & 0,726567 & 0,691595 & 0,608808 & 2,37251 & 2,357593 & 2,07136 \\
\hline 30 & 1,95421 & 1,97858 & 1,87385 & 0,485277 & 0,496762 & 0,481202 & 2,14177 & 2,093442 & 2,31998 \\
\hline 40 & 0,050796 & 0,026401 & 0,150149 & 0,824117 & 0,810774 & 0,733318 & 4,24035 & 4,276586 & 4,52993 \\
\hline 50 & 1,95623 & 1,978836 & 1,82829 & 1,1062 & 1,102023 & 1,14669 & 0,75352 & 0,660371 & 1,42154 \\
\hline
\end{tabular}


Çizelge 3. Yoğunluğu ve elastisite modülü üstel formda değişen çubuk için $P_{3}(\tau)$ yükü altında TFM ile bulunan serbest uçtaki deplasman değerlerinin analitik ve ANSYS sonuçları ile karşılaştırılması

\begin{tabular}{|c|c|c|c|c|c|c|c|c|c|}
\hline \multicolumn{10}{|c|}{$v(1, \tau)$} \\
\hline & \multicolumn{3}{|c|}{$a=0$} & \multicolumn{3}{|c|}{$a=1$} & \multicolumn{3}{|c|}{$a=2$} \\
\hline$\tau$ & TFM & Ref, [10] & ANSYS & TFM & Ref, [10] & ANSYS & TFM & Ref, [10] & ANSYS \\
\hline 5 & 0,697456 & 0,696882 & 0,694604 & 1,79934 & 1,800494 & 1,79658 & 3,45606 & 3,458083 & 3,45855 \\
\hline 10 & 1,10394 & 1,104459 & 1,09737 & 1,57887 & 1,57961 & 1,57023 & 3,5996 & 3,606126 & 3,59279 \\
\hline 20 & 0,89885 & 0,898296 & 0,903889 & 1,47445 & 1,471508 & 1,47719 & 2,72247 & 2,717392 & 2,72499 \\
\hline 30 & 1,10616 & 1,106679 & 1,09402 & 1,43238 & 1,431784 & 1,44200 & 3,62238 & 3,62549 & 3,65290 \\
\hline 40 & 0,898855 & 0,898302 & 0,909387 & 1,42607 & 1,424038 & 1,43205 & 3,03772 & 3,036661 & 3,05766 \\
\hline 50 & 1,10617 & 1,106679 & 1,10700 & 1,38319 & 1,3824 & 1,37667 & 3,10921 & 3,108574 & 3,16154 \\
\hline
\end{tabular}

Çizelge 4. Yoğunluğu ve elastisite modülü sinüzoidal formda değişen çubuk için $P_{1}(\tau)$ yükü altında TFM ile bulunan serbest uçtaki deplasman değerlerinin analitik ve ANSYS sonuçları ile karşılaştırılması

\begin{tabular}{|c|c|c|c|c|c|c|c|c|c|}
\hline \multicolumn{9}{|c|}{$\mathbf{a = 0}$} & \multicolumn{3}{c|}{$\mathbf{a = 1}$} \\
\hline & \multicolumn{3}{|c|}{$\mathbf{\tau})$} & \multicolumn{3}{c|}{ a=2 } \\
\hline $\boldsymbol{\tau}$ & TFM & Ref, [10] & ANSYS & TFM & Ref,[10] & ANSYS & TFM & Ref,[10] & ANSYS \\
\hline 5 & 3,01293 & 3,00649 & 3,00696 & 2,39753 & 2,39325 & 2,39293 & 7,69337 & 7,67786 & 7,72680 \\
\hline 10 & $-0,33030$ & $-0,33194$ & $-0,33121$ & $-0,25380$ & $-0,25524$ & $-0,25434$ & 0,01716 & 0,01721 & 0,01210 \\
\hline 20 & 0,25390 & 0,25144 & 0,24996 & 0,11608 & 0,11409 & 0,11281 & 0,56929 & 0,56217 & 0,56643 \\
\hline 30 & 0,15465 & 0,15091 & 0,15310 & 0,26976 & 0,26717 & 0,26870 & 1,24836 & 1,23844 & 1,24860 \\
\hline 40 & 0,93189 & 0,92725 & 0,92433 & 0,48438 & 0,48027 & 0,47920 & 2,15271 & 2,15268 & 2,15737 \\
\hline 50 & 0,97164 & 0,96584 & 0,96957 & 1,05025 & 1,04627 & 1,04647 & 3,3042 & 3,30424 & 3,30736 \\
\hline
\end{tabular}

Çizelge 5. Yoğunluğu ve elastisite modülü sinüzoidal formda değişen çubuk için $P_{2}(\tau)$ yükü altında TFM ile bulunan serbest uçtaki deplasman değerlerinin analitik ve ANSYS sonuçları ile karşılaştırılması

\begin{tabular}{|c|c|c|c|c|c|c|c|c|c|}
\hline \multicolumn{9}{|c|}{ a=0 } & \multicolumn{3}{c|}{ a=1 } & \multicolumn{3}{c|}{ a=2 } \\
\hline & \multicolumn{3}{|c|}{$\boldsymbol{\tau})$} \\
\hline $\boldsymbol{\tau}$ & TFM & Ref, 10] & ANSYS & TFM & Ref, [10] & ANSYS & TFM & Ref, [10] & ANSYS \\
\hline 5 & 1,41374 & 0,01998 & 1,38732 & 0,87905 & 0,87485 & 0,86046 & 4,2167 & 4,21988 & 4,51497 \\
\hline 10 & 2,78979 & 2,79598 & 2,68811 & 2,04846 & 2,05379 & 1,94477 & 4,92822 & 4,88116 & 4,92970 \\
\hline 20 & 0,04213 & 0,03728 & 0,16365 & 0,48546 & 0,47609 & 0,62242 & 3,98989 & 4,01468 & 4,05914 \\
\hline 30 & 2,79512 & 2,79598 & 2,64641 & 1,2719 & 1,27452 & 1,11143 & 4,28231 & 4,26753 & 4,04947 \\
\hline 40 & 0,03814 & 0,03728 & 0,21205 & 1,37882 & 1,36930 & 1,55770 & 4,45084 & 4,05653 & 3,97037 \\
\hline 50 & 2,8469 & 2,79598 & 2,58207 & 0,50381 & 0,51185 & 0,31635 & 3,05324 & 2,73448 & 2,73945 \\
\hline
\end{tabular}

Çizelge 6. Yoğunluğu ve elastisite modülü sinüzoidal formda değişen çubuk için $P_{3}(\tau)$ yükü altında TFM ile bulunan serbest uçtaki deplasman değerlerinin analitik ve ANSYS sonuçları ile karşılaştırılması

\begin{tabular}{|c|c|c|c|c|c|c|c|c|c|}
\hline \multicolumn{9}{|c|}{$\mathbf{a = 0}$} & \multicolumn{3}{c|}{$\mathbf{a = 1}$} & \multicolumn{3}{c|}{ a=2 } \\
\hline & \multicolumn{3}{|c|}{$\boldsymbol{c})$} \\
\hline $\boldsymbol{\tau}$ & TFM & Ref, [10] & ANSYS & TFM & Ref, [10] & ANSYS & TFM & Ref, [10] & ANSYS \\
\hline 5 & 0,98418 & 0,98111 & 0,980977 & 0,74643 & 0,74293 & 0,743068 & 3,86414 & 3,86569 & 3,88552 \\
\hline 10 & 1,55978 & 1,55729 & 1,54980 & 1,0515 & 1,04811 & 1,04349 & 3,8405 & 3,84217 & 3,84505 \\
\hline 20 & 1,26861 & 1,26415 & 1,27655 & 1,30635 & 1,30461 & 1,31007 & 4,01908 & 4,01898 & 4,01433 \\
\hline 30 & 1,56299 & 1,56038 & 1,54507 & 0,79908 & 0,79544 & 0,791522 & 3,58918 & 3,59032 & 3,63378 \\
\hline 40 & 1,26892 & 1,26420 & 1,28431 & 1,42204 & 1,42025 & 1,42323 & 4,01313 & 4,01696 & 4,02497 \\
\hline 50 & 1,56353 & 1,56033 & 1,53819 & 0,85374 & 0,85033 & 0,850061 & 3,68764 & 3,65701 & 3,68520 \\
\hline
\end{tabular}


Çizelge 7. Yoğunluğu ve elastisite modülü polinom formda değişen çubuk için $P_{1}(\tau)$ yükü altında TFM ile bulunan serbest uçtaki deplasman değerlerinin analitik ve ANSYS sonuçları ile karşılaştırılması

\begin{tabular}{|c|c|c|c|c|c|c|c|c|c|}
\hline \multicolumn{9}{|c|}{ a=0 } & \multicolumn{3}{c|}{$\mathbf{a = 1}$} & \multicolumn{3}{c|}{ a=2 } \\
\hline & \multicolumn{3}{|c|}{$\boldsymbol{\tau})$} \\
\hline $\boldsymbol{\tau}$ & TFM & Ref, [10] & ANSYS & TFM & Ref, [10] & ANSYS & TFM & Ref, [10] & ANSYS \\
\hline 5 & 2,13338 & 2,133376 & 2,12915 & 1,30411 & 1,304106 & 1,30111 & 0,881667 & 0,881667 & 0,879087 \\
\hline 10 & $-0,23388$ & $-0,23388$ & $-0,23452$ & $-0,0371$ & $-0,0371$ & $-0,0373$ & $-0,36271$ & $-0,36271$ & $-0,36140$ \\
\hline 20 & 0,179785 & 0,179786 & 0,176996 & $-0,09914$ & $-0,09914$ & $-0,1002$ & 0,059314 & 0,059314 & 0,057668 \\
\hline 30 & 0,109509 & 0,109508 & 0,108413 & $-0,08487$ & $-0,08487$ & $-0,08609$ & $-0,15767$ & $-0,15767$ & $-0,15662$ \\
\hline 40 & 0,659848 & 0,659849 & 0,654500 & 0,07812 & 0,07812 & 0,077231 & 0,216753 & 0,216752 & 0,213320 \\
\hline 50 & 0,688 & 0,688 & 0,686527 & 0,374068 & 0,374067 & 0,372233 & 0,190823 & 0,190822 & 0,191549 \\
\hline
\end{tabular}

Çizelge 8. Yoğunluğu ve elastisite modülü polinom formda değişen çubuk için $P_{2}(\tau)$ yükü altında TFM ile bulunan serbest uçtaki deplasman değerlerinin analitik ve ANSYS sonuçları ile karşılaştırılması

\begin{tabular}{|c|c|c|c|c|c|c|c|c|c|}
\hline \multicolumn{9}{|c|}{$\mathbf{a = 0}$} & \multicolumn{3}{|c|}{$\mathbf{a = 1}$} & \multicolumn{3}{|c|}{$\mathbf{a = 2}$} \\
\hline & \multicolumn{3}{|c|}{$\mathbf{0}$} & $\mathbf{1}$ & \\
\hline $\boldsymbol{\tau}$ & TFM & Ref, $[\mathbf{1 0}]$ & ANSYS & TFM & Ref, $[\mathbf{1 0 ]}$ & ANSYS & TFM & Ref, [10] & ANSYS \\
\hline 5 & 0,999845 & 1,000407 & 0,982324 & 0,107887 & 0,107655 & 0,112122 & 0,301791 & 0,301887 & 0,306523 \\
\hline 10 & 1,97439 & 1,97858 & 1,90338 & 0,252835 & 0,253724 & 0,236228 & 0,64419 & 0,644694 & 0,632406 \\
\hline 20 & 0,030772 & 0,0264007 & 0,115879 & 0,616221 & 0,615039 & 0,647566 & 0,068131 & 0,067815 & 0,071693 \\
\hline 30 & 1,97673 & 1,97858 & 1,87385 & 0,892067 & 0,893377 & 0,896216 & 0,557082 & 0,557428 & 0,565088 \\
\hline 40 & 0,032116 & 0,026401 & 0,150149 & 0,910138 & 0,908685 & 0,882136 & 0,159587 & 0,159312 & 0,150591 \\
\hline 50 & 1,99163 & 1,978836 & 1,82829 & 0,578499 & 0,578521 & 0,585328 & 0,437142 & 0,43645 & 0,434744 \\
\hline
\end{tabular}

Çizelge 9. Yoğunluğu ve elastisite modülü polinom formda değişen çubuk için $P_{3}(\tau)$ yükü altında TFM ile bulunan serbest uçtaki deplasman değerlerinin analitik ve ANSYS sonuçları ile karşılaştırılması

\begin{tabular}{|c|c|c|c|c|c|c|c|c|c|}
\hline \multicolumn{10}{|c|}{$\mathbf{a}=\mathbf{0}(\mathbf{1}) \boldsymbol{\tau})$} \\
\hline & \multicolumn{3}{|c|}{$\mathbf{a}$} & \multicolumn{3}{c|}{ a=2 } & \\
\hline $\boldsymbol{\tau}$ & TFM & Ref, [10] & ANSYS & TFM & Ref, [10] & ANSYS & TFM & Ref, [10] & ANSYS \\
\hline 5 & 0,696886 & 0,696882 & 0,694604 & 0,479316 & 0,479316 & 0,477698 & 0,450853 & 0,450851 & 0,448833 \\
\hline 10 & 1,10445 & 1,104459 & 1,09737 & 0,584101 & 0,584096 & 0,582325 & 0,450136 & 0,450135 & 0,448745 \\
\hline 20 & 0,898295 & 0,898296 & 0,903889 & 0,705466 & 0,705475 & 0,702761 & 0,192374 & 0,192377 & 0,190641 \\
\hline 30 & 1,1067 & 1,106679 & 1,09402 & 0,659433 & 0,659431 & 0,659555 & 0,493461 & 0,493458 & 0,492180 \\
\hline 40 & 0,898239 & 0,898302 & 0,909387 & 0,494009 & 0,494003 & 0,490425 & 0,169128 & 0,169133 & 0,169414 \\
\hline 50 & 1,10715 & 1,106679 & 1,08915 & 0,337535 & 0,337593 & 0,335158 & 0,493381 & 0,493355 & 0,489925 \\
\hline
\end{tabular}

\section{SONUÇLAR}

Heterojen yapıya sahip bir çubuğun zorlanmış titreşim analizi Laplace dönüşüm yöntemi ile birlikte Tamamlayıcı fonksiyonlar metodu (TFM) kullanılarak yapılmıştır. Laplace dönüşüm yöntemi zamandan bağımsız sınır değer problemini eksenel koordinatlarda verirken daha sonra bu problem TFM tarafından çözülmüştür. TFM benzer problemleri başlanğıç-değer sistemine çevirerek literatürdeki herhangi bir sayısal yöntem ile çözülmesine izin vermektedir. Bu çalışmada beşinci dereceden Runge-Kutta metodu kullanılarak sayısal olarak çözülen denklem sistemi Durbin'in sayısal ters dönüşümünü kullanarak zaman uzayına dönüştürülmüştür. TFM kullanılarak bulunan sayısal sonuçlar, literatürdeki analitik sonuçlar ve ANSYS sonuçları ile karşılaştırılmıştır. Burada açıkça görülen odur ki, bazı durumlarda TFM ile çözümde, çubuğun 20 parçaya bölünerek çözüm elde edilmesi sonuçlarda virgülden sonra beş haneli hassasiyet sağlarken, aynı sonuç için ANSYS'te ihtiyaç duyulan bölme sayısı binli rakamlara çıkmaktadır. Bu yöntemle çözüm zamanı büyük oranda azalmaktadır. 
Tamamlayıcı fonksiyonlar metodunun çubuğun dinamik analizinde sağladı $\breve{g}$ avantajlar sırasıyla:

(1) Zorlanmış titreşim etkisi doğrudan elde edilir.

(2) Zorlanmış titreşim analizi için serbest titreşim frekanslarına ve mod şekillerine ihtiyaç yoktur.

(3) Çözüm yöntemi yoğunluk ve elastisite modülünü ifade eden belirli bazı özel fonksiyonlara bağımlı değildir, yoğunluk ve elastisite modülünü ifade eden tüm keyfi fonksiyonlar için son derece uygundur.

(4) Sonlu elemanlar yöntemi gibi diğer sayısal yöntemlerle karşılaştırıldığında, daha az sürede ve daha az maliyetle daha hassas sonuçlar bulunabilmektedir.

Bu çalışmada elde edilen sonuçlar, analitik olarak eldesi mümkün olmayan, elastisite modülü, yoğunluk ve kesit alanının birlikte bir eksen boyunca değişken olduğu çubukların gelecekteki titreşim analizi araştırmalarında bir referans oluşturacaktır.

\section{KAYNAKLAR}

1. Raj, A., Sujith, R.I., 2005. Closed-form Solutions for the Free Longitudinal Vibration of Inhomogeneous Rods, J. Sound. Vib. 283, 1015-1030.

2. Nachum, S., Altus, E., 2007. Natural Frequencies and Mode Shapes of Deterministic and Tochastic Non-homogeneous Rods and Beams, J. Sound. Vib., 302, 903-924.

3. Horgan, C.O., Chan, A.M., 1999. Vibration of Inhomogeneous Strings, Rods and Membranes, J. Sound. Vib. 225, 503-513.

4. Abrate , S., 1995. Vibration of Non-uniform Rods and Beams, Journal of Sound and Vibration. 185, 703-716.

5. Kumar, B.M., Sujith, R.I., 1997. Exact Solutions for the Longitudinal Vibration of Non-uniform Rods, Journal of Sound and Vibration, 207,5, 721-729.

6. Li, Q.S., 2000. Exact Solutions for Free Longitudinal Vibration of Non-uniform Rods, Journal of Sound and Vibration, 234, 1, 1-19.

7. Li, Q.S., 2000. Exact Solutions for free Longitudinal Vibration of Bars with Non- uniform Cross-section, Journal of Applied Mechanics and Engineering, 5, 3, 521-541.

8. Li, Q.S., 2000. Exact Solutions for Longitudinal Vibration of Multi-step Bars with Varying Cross-section, Journal of Vibration and Acoustics, 122, 183-187.

9. Celebi, K., Keles, I., Tutuncu, N., 2011. Exact Solutions for Forced Vibration of Non-uniform Rods by Laplace Transformation, Gazi University Journal of Science, 24,2, 347-353.

10. Celebi, K., Keles, I., Tutuncu, N., 2012. Closed-form Solutions for Forced Vibration Analysis of Inhomogeneous Rod, J. Fac. Eng. Archit. Gaz., 27, 4, 753-763.

11. Shokrollahi, M., Nejad, A.Z.B., 2014. Numerical Analysis of Free Longitudinal Vibration of Nonuniform Rods: Discrete Singular Convolution Approach, Journal of Engineering Mechanics, 140, 8.

12. Aktas, Z., 1972. Numerical Solutions of Twopoint Boundary Value Problems, METU, Department of Computer Eng., Ankara, Turkey.

13. Roberts, S.M., Shipman, J.S., 1979. Fundamental Matrix and Two-point BoundaryValue Problems, Journal of Optimization Theory and Application, 28, 1, 77-78.

14. Agarwal, R.P., 1982. On the Method of Complementary Functions for Nonlinear Boundary-value Problems, Journal of Optimization Theory and Applications, 36, 1, 139-144.

15. Y1ldirım, V., 1997. Free Vibration Analysis of Non-cylindrical Coil Springs by Combined use of the Transfer Matrix and the Complementary Functions Methods, Communications in Numerical Methods in Engineering, 13, 487-494.

16. Calim, F.F., 2009. Free and Forced Vibration of Non-uniform Composite Beams, Composite Structures, 88, 413-423.

17. Tütüncü, N., Temel B., 2009. A Novel Approach to Stress Analysis of Pressurized FGM Cylinders, Disks and Spheres, Composite Structure, 91, 385-390.

18. Durbin, F., 1974. Numerical Inversion of Laplace Transforms: an Efficient Improvement to Dubner and Abate's Method, The Computer Journal, 17, 371-376. 
